\title{
STUDY OF THREE DIMENSIONAL PROPAGATION OF WAVES IN HOLLOW POROELASTIC CIRCULAR CYLINDERS
}

\author{
S.A. SHAH \\ Department of Mathematics \\ Deccan College of Engineering and Technology \\ Hyderabad-500 001 (T.S) INDIA \\ E-mail:ahmed_shah67@yahoo.com
}

\begin{abstract}
Employing Biot's theory of wave propagation in liquid saturated porous media, waves propagating in a hollow poroelastic circular cylinder of infinite extent are investigated. General frequency equations for propagation of waves are obtained each for a pervious and an impervious surface. Degenerate cases of the general frequency equations of pervious and impervious surfaces, when the longitudinal wavenumber $k$ and angular wavenumber $n$ are zero, are considered. When $k=0$, the plane-strain vibrations and longitudinal shear vibrations are uncoupled and when $k \neq 0$ these are coupled. It is seen that the frequency equation of longitudinal shear vibrations is independent of the nature of the surface. When the angular (or circumferential) wavenumber is zero, i.e., $n=0$, axially symmetric vibrations and torsional vibrations are uncoupled. For $n \neq 0$ these vibrations are coupled. The frequency equation of torsional vibrations is independent of the nature of the surface. By ignoring liquid effects, the results of a purely elastic solid are obtained as a special case.
\end{abstract}

Key words: Biot's theory, poroelastic cylindrical shell, pervious surface, impervious surface, wavenumber, frequency.

\section{Introduction}

Free vibrations of a solid cylinder of isotropic elastic material were given by Love (1944). Gazis (1959) discussed the propagation of free harmonic waves along a hollow elastic circular cylinder of infinite extent. Employing Biot's (1956) theory, Tajuddin and Sarma (1980) studied the torsional vibrations of poroelastic cylinders. Wisse et al. (2002) presented the experimental results of guided wave modes in porous cylinders. Chao et al. (2004) studied the shock-induced borehole waves in porous formations. The model consisting of concentric cylinders having different saturation levels is used to study the propagation of waves in porous cylinders by Berryman and Pride (2005) who obtained two distinct analytical expressions for torsional wave modes. Farhang et al. (2007) investigated the wave propagation in transversely isotropic cylinders. Sharma and Sharma (2010) studied the three dimensional free vibrations of transradially thermoelastic spheres. Karpfinger et al. (2010) used the method of discretization of cylindrical structures along the radial axis to study the dispersion properties of Stonely waves and attenuation in poroelastic layers. The author $(2006 ; 2007 ; 2008 ; 2010 \mathrm{a} ; 2010 \mathrm{~b} ; 2011)$ studied different problems wave propagation in poroelastic cylinders.

In the present analysis, the free vibrations of an infinite hollow poroelastic circular cylinder are studied employing general displacement components in cylindrical polar coordinates, following Biot's (1956) theory. General frequency equations of pervious and impervious surfaces are obtained. Let the infinite poroelastic hollow cylinder be homogeneous and isotropic. Degenerate cases of the general frequency equations of pervious and impervious surfaces, when the longitudinal wavenumber $k$ and angular wavenumber $\mathrm{n}$ are zero, are considered. When $k=0$, the plane-strain vibrations and longitudinal shear vibrations are uncoupled and when $k \neq 0$ these are coupled. When the angular (or circumferential) wavenumber is zero, i.e., $n=0$, axially symmetric vibrations and torsional vibrations are uncoupled. For $n \neq 0$ 
these vibrations are coupled. By ignoring liquid effects, the results of a purely elastic solid are obtained as a special case. The plots of non-dimensional frequency as a function of ratio of wall thickness to wavelength are presented for non-axially symmetric vibrations each for a pervious and an impervious surface. Results are discussed for two different poroelastic materials namely, sandstone saturated with kerosene (Fatt, 1959) and sandstone saturated with water (Yew and Jogi, 1976). It is seen that frequency is same for $n=1$ and 2 in the case of a thin poroelastic cylindrical shell each for a pervious and an impervious surface. Frequency is not the same, however, for a thick shell or a poroelastic solid cylinder.

The considered problem is of importance in civil engineering, ceramic industry where the frequency play an important role. The investigation is also applicable to Bio-Mechanics, wherein osseous tissue, bony elements saturated with a fluid are approximated by a hollow poroelastic cylinder.

\section{Governing equations}

The equations of motion of a homogeneous, isotropic poroelastic solid (Biot, 1956) in the presence of dissipation $b$ are

$$
\begin{aligned}
& N \nabla^{2} \boldsymbol{u}+(A+N) \nabla e+Q \nabla \in=\frac{\partial^{2}}{\partial t^{2}}\left(\rho_{11} \boldsymbol{u}+\rho_{12} \boldsymbol{U}\right)+b \frac{\partial}{\partial t}(\boldsymbol{u}-\boldsymbol{U}), \\
& Q \nabla e+R \nabla \in=\frac{\partial^{2}}{\partial t^{2}}\left(\rho_{12} \boldsymbol{u}+\rho_{22} \boldsymbol{U}\right)-b \frac{\partial}{\partial t}(\boldsymbol{u}-\boldsymbol{U}),
\end{aligned}
$$

here $\nabla^{2}$ is the Laplacian operator, $\boldsymbol{u}=(u, v, w)$ and $\boldsymbol{U}=(U, V, W)$ are displacements of the solid and liquid respectively, $e$ and $\in$ are the dilatations of the solid and liquid, $A, N, Q, R$ are all poroelastic constants and $\rho_{i j}(i, j=1,2)$ are the mass coefficients following Biot (1956).

The stresses $\sigma_{i j}$, and the liquid pressure s are

$$
\begin{aligned}
& \sigma_{i j}=2 N e_{i j}+(A e+Q \in) \delta_{i j}, \quad(i, j=r, \theta, z), \\
& s=Q e+R \in
\end{aligned}
$$

where $\delta_{i j}$ is the well-known Kronecker delta function.

\section{Solution of the problem}

Let $(r, \theta, z)$ be the cylindrical polar coordinates. Consider a homogeneous, isotropic, infinite hollow poroelastic cylinder with inner and outer radii $r_{1}$ and $r_{2}$, respectively, having thickness $h\left[=\left(r_{2}-r_{l}\right)>0\right]$ whose axis is in the direction of the $z$-axis.

Let

$$
\boldsymbol{u}=\nabla \Phi_{1}+\nabla \times \boldsymbol{\Psi}_{1}, \quad \boldsymbol{U}=\nabla \Phi_{2}+\nabla \times \boldsymbol{\Psi}_{2}
$$

In Eq.(3.1), $\boldsymbol{u}, \boldsymbol{U}$ are displacements of the solid and liquid, respectively, and $\Phi_{1}, \Phi_{2}$ are scalar potentials, $\boldsymbol{\Psi}_{1}, \boldsymbol{\Psi}_{2}$ are vector potentials.

Let

$$
\boldsymbol{\Psi}_{1}=\left(h_{r}, h_{\theta}, h_{z}\right), \quad \boldsymbol{\Psi}_{2}=\left(H_{r}, H_{\theta}, H_{z}\right) .
$$

Equations (2.1), together with Eqs (3.1) reduce to 


$$
\begin{aligned}
& P \nabla^{2} \Phi_{1}+Q \nabla^{2} \Phi_{2}=\left(\rho_{11} \ddot{\Phi}_{1}+\rho_{12} \ddot{\Phi}_{2}\right)+b\left(\dot{\Phi}_{1}-\dot{\Phi}_{2}\right) \\
& Q \nabla^{2} \Phi_{1}+R \nabla^{2} \Phi_{2}=\left(\rho_{12} \ddot{\Phi}_{1}+\rho_{22} \ddot{\Phi}_{2}\right)-b\left(\dot{\Phi}_{1}-\dot{\Phi}_{2}\right) \\
& N \nabla^{2} \boldsymbol{\Psi}_{1}=\left(\rho_{11} \ddot{\mathbf{\Psi}}_{1}+\rho_{12} \ddot{\mathbf{\Psi}}_{2}\right)+b\left(\dot{\boldsymbol{\Psi}}_{1}-\dot{\boldsymbol{\Psi}}_{2}\right), \\
& 0=\left(\rho_{12} \ddot{\mathbf{\Psi}}_{1}+\rho_{22} \ddot{\mathbf{\Psi}}_{2}\right)+b\left(\dot{\boldsymbol{\Psi}}_{1}-\dot{\boldsymbol{\Psi}}_{2}\right)
\end{aligned}
$$

where a dot over a quantity represents differentiation with respect to time $t$ and $P=A+2 N$.

Let

$$
\begin{aligned}
& \Phi_{l}=f_{l}(r) \cos (n \theta) e^{i(k z+\omega t)}, \quad \Phi_{2}=f_{2}(r) \cos (n \theta) e^{i(k z+\omega t)}, \\
& h_{r}=g_{r}(r) \sin (n \theta) e^{i(k z+\omega t)}, \quad h_{\theta}=g_{\theta}(r) \cos (n \theta) e^{i(k z+\omega t)}, \quad h_{z}=g_{3}(r) \sin (n \theta) e^{i(k z+\omega t),} \\
& H_{r}=G_{r}(r) \sin (n \theta) e^{i(k z+\omega t)}, H_{\theta}=G_{\theta}(r) \cos (n \theta) e^{i(k z+\omega t)}, H_{z}=G_{3}(r) \sin (n \theta) e^{i(k z+\omega t) .} .
\end{aligned}
$$

In Eqs (3.4), $k$ is the axial wavenumber, $n$ is the integer number of waves around the circumference or also known as the angular wavenumber, $\omega$ is circular frequency. The second and third Eqs of (3.3) with (3.2), when first two Eqs of (3.3) remain the same, are reduced to

$$
\begin{aligned}
& P \nabla^{2} \Phi_{1}+Q \nabla^{2} \Phi_{2}=\left(\rho_{11} \ddot{\Phi}_{1}+\rho_{12} \ddot{\Phi}_{2}\right)+b\left(\dot{\Phi}_{1}-\dot{\Phi}_{2}\right), \\
& Q \nabla^{2} \Phi_{1}+R \nabla^{2} \Phi_{2}=\left(\rho_{12} \ddot{\Phi}_{1}+\rho_{22} \ddot{\Phi}_{2}\right)-b\left(\dot{\Phi}_{1}-\dot{\Phi}_{2}\right), \\
& N\left(\nabla^{2} h_{r}-\frac{h_{r}}{r^{2}}-\frac{2}{r^{2}} \frac{\partial h_{\theta}}{\partial \theta}\right)=\left(\rho_{11} \ddot{h}_{r}+\rho_{12} \ddot{H}_{r}\right)+b\left(\dot{h}_{r}-\dot{H}_{r}\right), \\
& N\left(\nabla^{2} h_{\theta}-\frac{h_{\theta}}{r^{2}}+\frac{2}{r^{2}} \frac{\partial h_{r}}{\partial \theta}\right)=\left(\rho_{11} \ddot{h}_{\theta}+\rho_{12} \ddot{H}_{\theta}\right)+b\left(\dot{h}_{\theta}-\dot{H}_{\theta}\right), \\
& N \nabla^{2} h_{z}=\left(\rho_{11} \ddot{h}_{z}+\rho_{12} \ddot{H}_{z}\right)+\left(\dot{h}_{z}-\dot{H}_{z}\right), \\
& 0=\left(\rho_{12} \ddot{h}_{r}+\rho_{22} \ddot{H}_{r}\right)-b\left(\dot{h}_{r}-\dot{H}_{r}\right), \\
& 0=\left(\rho_{12} \ddot{h}_{\theta}+\rho_{22} \ddot{H}_{\theta}\right)-b\left(\dot{h}_{\theta}-\dot{H}_{\theta}\right), \\
& 0=\left(\rho_{12} \ddot{h}_{z}+\rho_{22} \ddot{H}_{z}\right)-b\left(\dot{h}_{z}-\dot{H}_{z}\right) .
\end{aligned}
$$

Equations (3.5) with the help of Eqs (3.4) reduce to 


$$
\begin{aligned}
& P \Delta f_{1}+Q \Delta f_{2}=-\omega^{2}\left[K_{11} f_{1}+K_{12} f_{2}\right], \\
& Q \Delta f_{1}+R \Delta f_{2}=-\omega^{2}\left[K_{12} f_{1}+K_{22} f_{2}\right], \\
& N\left[\Delta g_{r}-\frac{g_{r}}{r^{2}}+\frac{2 n g_{\theta}}{r^{2}}\right]=-\omega^{2}\left[K_{11} g_{r}+K_{12} G_{r}\right], \\
& N\left[\Delta g_{\theta}-\frac{g_{\theta}}{r^{2}}+\frac{2 n g_{r}}{r^{2}}\right]=-\omega^{2}\left[K_{11} g_{\theta}+K_{12} G_{\theta}\right], \\
& N \Delta g_{3}=-\omega^{2}\left[K_{11} g_{3}+K_{12} G_{3}\right], \\
& 0=-\omega^{2}\left[K_{12} g_{r}+K_{22} G_{r}\right], \\
& 0=-\omega^{2}\left[K_{12} g_{\theta}+K_{22} G_{\theta}\right], \\
& 0=-\omega^{2}\left[K_{12} g_{3}+K_{22} G_{3}\right]
\end{aligned}
$$

where

$$
\begin{aligned}
& \Delta=\frac{d^{2}}{d r^{2}}+\frac{1}{r} \frac{d}{d r}-\frac{n^{2}}{r^{2}}-k^{2}, \\
& K_{11}=\rho_{11}-\frac{i b}{\omega}, \quad K_{12}=\rho_{12}+\frac{i b}{\omega}, \quad K_{22}=\rho_{22}-\frac{i b}{\omega} .
\end{aligned}
$$

The general solutions of Eqs (3.6) can be obtained in terms of the Bessel functions of the first and second kind $J$ and $Y$ or the modified the Bessel functions of first and second kind $I$ and $K$, depending on whether its arguments $\alpha_{1} r, \alpha_{2} r, \alpha_{3} r$ are real or imaginary. Equation (3.6) after a long calculation yields

$$
\begin{aligned}
& f_{l}(r)=C_{1} Z_{n}\left(\alpha_{1} r\right)+C_{2} W_{n}\left(\alpha_{1} r\right)+C_{3} Z_{n}\left(\alpha_{2} r\right)+C_{4} W_{n}\left(\alpha_{2} r\right), \\
& g_{3}(r)=A_{3} Z_{n}\left(\alpha_{3} r\right)+B_{3} W_{n}\left(\alpha_{3} r\right), \\
& 2 g_{1}(r)=g_{r}-g_{\theta}=2 A_{1} Z_{n+l}\left(\alpha_{3} r\right)+2 B_{1} W_{n+l}\left(\alpha_{3} r\right), \\
& 2 g_{2}(r)=g_{r}+g_{\theta}=2 A_{2} Z_{n-1}\left(\alpha_{3} r\right)+2 B_{2} W_{n-l}\left(\alpha_{3} r\right),
\end{aligned}
$$

where $Z_{n}$ denotes Bessel function or modified Bessel function of first kind, i.e., $J_{n}$ or $I_{n}$ and $W_{n}$ denotes the Bessel function or modified Bessel function of second kind, i.e., $Y_{n}$ or $K_{n}$, depending on its arguments

$$
\alpha_{1}^{2}=\xi_{1}^{2}-k^{2}, \quad \alpha_{2}^{2}=\xi_{2}^{2}-k^{2}, \quad \alpha_{3}^{2}=\xi_{3}^{2}-k^{2},
$$

are positive or negative and 


$$
\omega^{2}=\xi_{i}^{2} V_{i}^{2} \quad(i=1,2,3)
$$

In Eq.(3.10) $V_{1}, V_{2}$ are dilatational wave velocities of first kind and second kind respectively, and $V_{3}$ is shear wave velocity.

The gauge invariance property (Gazis, 1959) is used to eliminate two integration constants from Eq.(3.8). Any one of the potential functions $g_{1}, g_{2}$ or $g_{3}$ can be set equal to zero, without loss of generality of solution. Choosing $g_{2}=0$, we obtain

$$
g_{r}=-g_{\theta}=g_{1}
$$

The displacement vector of the solid $\boldsymbol{u}=(u, v, w)$ with the help of Eqs (3.1), (3.2) is given by

$$
u=\frac{\partial \Phi_{1}}{\partial r}+\frac{1}{r} \frac{\partial h_{z}}{\partial \theta}-\frac{\partial h_{\theta}}{\partial z}, \quad v=\frac{1}{r} \frac{\partial \Phi_{1}}{\partial \theta}+\frac{\partial h_{r}}{\partial z}-\frac{\partial h_{z}}{\partial r}, \quad w=\frac{\partial \Phi_{1}}{\partial z}+\frac{\partial h_{\theta}}{\partial r}+\frac{h_{\theta}}{r}-\frac{1}{r} \frac{\partial h_{r}}{\partial \theta} .
$$

Substituting Eqs (3.4) in Eqs (3.12), the displacement components of the solid are

$$
\begin{aligned}
& u=\left(f_{l}^{\prime}+\frac{n}{r} g_{3}-i k g_{\theta}\right) \cos (n \theta) e^{i(k z+\omega t)}, \quad v=\left(-\frac{n}{r} f_{l}+i k g_{r}-g_{3}^{\prime}\right) \sin (n \theta) e^{i(k z+\omega t)}, \\
& w=\left(i k f_{l}+g_{\theta}^{\prime}+g_{\theta}-\frac{n}{r} g_{r}\right) \cos (n \theta) e^{i(k z+\omega t)},
\end{aligned}
$$

Substituting Eq.(3.11) in Eqs (3.13), the solid displacement is

$$
\begin{aligned}
& u=\left(f_{1}^{\prime}+\frac{n}{r} g_{3}+i k g_{1}\right) \cos (n \theta) e^{i(k z+\omega t)}, \quad v=\left(-\frac{n}{r} f_{1}+i k g_{1}-g_{3}^{\prime}\right) \sin (n \theta) e^{i(k z+\omega t)}, \\
& w=\left(i k f_{1}-g_{1}^{\prime}-\frac{(n+l)}{r} g_{1}\right) \cos (n \theta) e^{i(k z+\omega t)}
\end{aligned}
$$

where the 'prime' over a quantity denotes differentiation with respect to $r$.

The proper selection of either the Bessel function or modified Bessel function is shown in Tab.1.

Table 1

\begin{tabular}{|c|c|}
\hline Interval & Functions used \\
\hline$\left|k V_{l}\right|<\omega$ & $J\left(\alpha_{1} r\right), Y\left(\alpha_{l} r\right), J\left(\alpha_{2} r\right), Y\left(\alpha_{2} r\right), J\left(\alpha_{3} r\right), Y\left(\alpha_{3} r\right)$. \\
\hline$\left|k V_{3}\right|<\omega<\left|k V_{l}\right|$ & $I\left(\beta_{1} r\right), K\left(\beta_{1} r\right), I\left(\beta_{2} r\right), K\left(\beta_{2} r\right), J\left(\alpha_{3} r\right), Y\left(\alpha_{3} r\right)$. \\
\hline$\omega<\left|k V_{l}\right|$ & $I\left(\beta_{1} r\right), K\left(\beta_{1} r\right), I\left(\beta_{2} r\right), K\left(\beta_{2} r\right), I\left(\beta_{3} r\right), K\left(\beta_{3} r\right)$. \\
\hline
\end{tabular}


$\beta_{1}, \beta_{2}, \beta_{3}$, are the absolute values of $\alpha_{1}, \alpha_{2}, \alpha_{3}$, respectively, when these values are imaginary, and $\alpha_{1}, \alpha_{2}, \alpha_{3}$ are defined in Eq.(3.9).

The dilatations of the solid and liquid media are

$$
e=\Delta f_{1}(r) \cos (n \theta) e^{i(k z+\omega t)}, \quad \in=\Delta f_{2}(r) \cos (n \theta) e^{i(k z+\omega t)}
$$

where $\Delta$ is defined in Eq.(3.7).

By substituting Eqs (3.14) into strain displacement relations and then using Eq.(2.2), the stresses $\sigma_{i j}$ and the liquid pressure $s$ are

$$
\begin{aligned}
& \sigma_{r r}+s=\left[C_{1} M_{11}(r)+C_{2} M_{12}(r)+C_{3} M_{13}(r)+C_{4} M_{14}(r)+\right. \\
& \left.+A_{3} M_{15}(r)+B_{3} M_{16}(r)+A_{1} M_{17}(r)+B_{1} M_{18}(r)\right] \cos (n \theta) e^{i(k z+\omega t)}, \\
& \sigma_{r \theta}=\left[C_{1} M_{21}(r)+C_{2} M_{22}(r)+C_{3} M_{23}(r)+C_{4} M_{24}(r)+\right. \\
& \left.+A_{3} M_{25}(r)+B_{3} M_{26}(r)+A_{1} M_{27}(r)+B_{1} M_{28}(r)\right] \sin (n \theta) e^{i(k z+\omega t)}, \\
& \sigma_{r z}=\left[C_{1} M_{31}(r)+C_{2} M_{32}(r)+C_{3} M_{33}(r)+C_{4} M_{34}(r)+\right. \\
& \left.+A_{3} M_{35}(r)+B_{3} M_{36}(r)+A_{1} M_{37}(r)+B_{1} M_{38}(r)\right] \cos (n \theta) e^{i(k z+\omega t)}, \\
& s=\left[C_{1} M_{41}(r)+C_{2} M_{42}(r)+C_{3} M_{43}(r)+C_{4} M_{44}(r)\right] \cos (n \theta) e^{i(k z+\omega t)}, \\
& \frac{\partial s}{\partial r}=\left[C_{1} N_{41}(r)+C_{2} N_{42}(r)+C_{3} N_{43}(r)+C_{4} N_{44}(r)\right] \cos (n \theta) e^{i(k z+\omega t),}
\end{aligned}
$$

where the coefficients $M_{i j}(r)$ and $N_{i j}(r)$ are given as

$$
\begin{aligned}
& M_{11}(r)=\left\{2 N\left[\frac{n(n-1)}{r^{2}}-\lambda_{1} \alpha_{1}^{2}\right]+\right. \\
& \left.+\left[(Q+R) \delta_{1}^{2}-(A+Q)\right]\left(k^{2}+\lambda_{1} \alpha_{1}^{2}\right)\right\} Z_{n}\left(\beta_{I} r\right)+\frac{2 \lambda_{I} N \beta_{1}}{r} Z_{n+1}\left(\beta_{I} r\right), \\
& M_{12}(r)=\left\{2 N\left[\frac{n(n-1)}{r^{2}}-\lambda_{I} \alpha_{1}^{2}\right]+\right. \\
& \left.+\left[(Q+R) \delta_{1}^{2}-(A+Q)\right]\left(k^{2}+\lambda_{I} \alpha_{1}^{2}\right)\right\} W_{n}\left(\beta_{1} r\right)+\frac{2 N \beta_{1}}{r} Z_{n+1}\left(\beta_{1} r\right), \\
& M_{13}(r)=\left\{2 N\left[\frac{n(n-1)}{r^{2}}-\lambda_{1} \alpha_{2}^{2}\right]+\right. \\
& \left.+\left[(Q+R) \delta_{2}^{2}-(A+Q)\right]\left(k^{2}+\lambda_{I} \alpha_{2}^{2}\right)\right\} Z_{n}\left(\beta_{2} r\right)+\frac{2 \lambda_{1} N \beta_{2}}{r} Z_{n+l}\left(\beta_{2} r\right),
\end{aligned}
$$




$$
\begin{aligned}
& M_{14}(r)=\left\{2 N\left[\frac{n(n-1)}{r^{2}}-\lambda_{1} \alpha_{2}^{2}\right]+\right. \\
& \left.+\left[(Q+R) \delta_{2}^{2}-(A+Q)\right]\left(k^{2}+\lambda_{1} \alpha_{2}^{2}\right)\right\} W_{n}\left(\beta_{2} r\right)+\frac{2 N \beta_{2}}{r} Z_{n+1}\left(\beta_{2} r\right) \\
& M_{15}(r)=\frac{2 N n(n-1)}{r^{2}} Z_{n}\left(\beta_{3} r\right)-\frac{2 \lambda_{2} N n \beta_{3}}{r} Z_{n+1}\left(\beta_{3} r\right) \\
& M_{16}(r)=\frac{2 N n(n-1)}{r^{2}} W_{n}\left(\beta_{3} r\right)-\frac{2 N n \beta_{3}}{r} Z_{n+1}\left(\beta_{3} r\right) \\
& M_{17}(r)=2 N i k \beta_{3} Z_{n}\left(\beta_{3} r\right)-\frac{2 N(n+1) i k}{r} Z_{n+1}\left(\beta_{3} r\right) \\
& M_{18}(r)=2 \lambda{ }_{2} N i k \beta_{3} W_{n}\left(\beta_{3} r\right)-\frac{2 N(n+1) i k}{r} W_{n+1}\left(\beta_{3} r\right) \\
& M_{28}(r)=\lambda(r)=\frac{2 N n i k}{r} Z_{n}\left(\beta_{1} r\right)-2 \lambda_{1} N i k \beta_{1} Z_{n+1}\left(\beta_{1} r\right) \\
& M_{27}(r)=i k N \beta_{3} Z_{n}\left(\beta_{3} r\right)-\frac{2 N(n+1) i k}{r} Z_{n+1}\left(\beta_{3} r\right) \\
& M_{25}(r)=\left[\frac{2 N n(1-n)}{r^{2}}+\lambda_{2} N \alpha_{3}^{2}\right] Z_{n}\left(\beta_{3} r\right)-\frac{2 N(n)}{r} Z_{n}\left(\beta_{1} r\right)+\frac{2 \lambda{ }_{1} N n \beta_{1}}{r} Z_{n+1}\left(\beta_{1} r\right) \\
& M_{22}(r)=\frac{2 N n(1-n)}{r^{2}} W_{n}\left(\beta_{1} r\right)+\frac{2 N n \beta_{1}}{r} W_{n+1}\left(\beta_{1} r\right) \\
& M_{23}(r)=\frac{2 N n(1-n)}{r^{2}} Z_{n}\left(\beta_{2} r\right)+\frac{2 \lambda_{1} N n \beta_{2}}{r} Z_{n+1}\left(\beta_{2} r\right) \\
& \left.M_{n} r\right)
\end{aligned}
$$




$$
\begin{aligned}
& M_{32}(r)=\frac{2 N n i k}{r} W_{n}\left(\beta_{1} r\right)-2 N i k \beta_{1} W_{n+1}\left(\beta_{1} r\right), \\
& M_{33}(r)=\frac{2 N n i k}{r} Z_{n}\left(\beta_{2} r\right)-2 \lambda_{1} N i k \beta_{2} Z_{n+1}\left(\beta_{2} r\right), \\
& M_{34}(r)=\frac{2 N n i k}{r} W_{n}\left(\beta_{2} r\right)-2 N i k \beta_{2} W_{n+1}\left(\beta_{2} r\right), \\
& M_{35}(r)=\frac{N n i k}{r} Z_{n}\left(\beta_{3} r\right), \quad M_{36}(r)=\frac{N n i k}{r} W_{n}\left(\beta_{3} r\right), \\
& M_{37}(r)=\frac{-N n \beta_{3}}{r} Z_{n}\left(\beta_{3} r\right)-N\left(k^{2}-\lambda_{2} \alpha_{3}^{2}\right) Z_{n+1}\left(\beta_{3} r\right), \\
& M_{38}(r)=\frac{-\lambda_{2} N n \beta_{3}}{r} W_{n}\left(\beta_{3} r\right)-N\left(k^{2}-\lambda_{2} \alpha_{3}^{2}\right) Z_{n+1}\left(\beta_{3} r\right), \\
& M_{41}(r)=\left(R \delta_{1}^{2}-Q\right)\left(k^{2}+\lambda_{1} \alpha_{1}^{2}\right) Z_{n}\left(\beta_{1} r\right), \quad M_{42}(r)=\left(R \delta_{1}^{2}-Q\right)\left(k^{2}+\lambda_{1} \alpha_{l}^{2}\right) W_{n}\left(\beta_{1} r\right), \\
& M_{43}(r)=\left(R \delta_{2}^{2}-Q\right)\left(k^{2}+\lambda_{1} \alpha_{2}^{2}\right) Z_{n}\left(\beta_{2} r\right), \quad M_{44}(r)=\left(R \delta_{2}^{2}-Q\right)\left(k^{2}+\lambda_{1} \alpha_{2}^{2}\right) W_{n}\left(\beta_{2} r\right), \\
& N_{4 l}(r)=\frac{n}{r}\left(R \delta_{I}^{2}-Q\right)\left(k^{2}+\lambda_{l} \alpha_{l}^{2}\right) Z_{n}\left(\beta_{I} r\right)-\beta_{I}\left(R \delta_{l}^{2}-Q\right)\left(\alpha_{I}^{2}+\lambda_{l} k^{2}\right) Z_{n+l}\left(\beta_{I} r\right), \\
& N_{42}(r)=\frac{n}{r}\left(R \delta_{1}^{2}-Q\right)\left(k^{2}+\lambda_{I} \alpha_{1}^{2}\right) W_{n}\left(\beta_{1} r\right)-\beta_{I}\left(R \delta_{I}^{2}-Q\right)\left(\lambda_{I} \alpha_{I}^{2}+k^{2}\right) W_{n+1}\left(\beta_{I} r\right), \\
& N_{43}(r)=\frac{n}{r}\left(R \delta_{2}^{2}-Q\right)\left(k^{2}+\lambda_{1} \alpha_{2}^{2}\right) Z_{n}\left(\beta_{2} r\right)-\beta_{2}\left(R \delta_{2}^{2}-Q\right)\left(\alpha_{2}^{2}+\lambda_{1} k^{2}\right) Z_{n+1}\left(\beta_{2} r\right), \\
& N_{44}(r)=\frac{n}{r}\left(R \delta_{2}^{2}-Q\right)\left(k^{2}+\lambda_{1} \alpha_{2}^{2}\right) W_{n}\left(\beta_{2} r\right)-\beta_{2}\left(R \delta_{2}^{2}-Q\right)\left(\lambda_{1} \alpha_{2}^{2}+k^{2}\right) W_{n+1}\left(\beta_{2} r\right),
\end{aligned}
$$

In Eqs (3.21),

$$
\delta_{i}^{2}=\frac{1}{\left(R K_{12}-Q K_{22}\right)}\left[\left(R K_{11}-Q K_{12}\right)-V_{i}^{-2}\left(P R-Q^{2}\right)\right] . \quad(i=1,2)
$$

Also $\lambda_{1}$ and $\lambda_{2}$ are parameters which are introduced in order to account for the differences in the recurrence and differentiation formulas between the different kinds of Bessel functions. The values of these parameters are 1 and -1 when Bessel functions of first kind and second kind and modified Bessel functions of first and second kind are used. It is seen from Table-I that $\lambda_{1}$ and $\lambda_{2}$ vary as follows 


$$
\begin{array}{lcr}
\left|k V_{1}\right|<\omega: & \lambda_{1}=1, & \lambda_{2}=1, \\
\left|k V_{3}\right|<\omega<\left|k V_{1}\right|: & \lambda_{1}=-1, & \lambda_{2}=1, \\
\omega<\left|k V_{1}\right|: & \lambda_{1}=-1, & \lambda_{2}=-1 .
\end{array}
$$

\section{Boundary conditions - frequency equation}

The boundary conditions for traction free inner and outer surfaces of the hollow poroelastic cylinder in the case of a pervious surface are

$$
\sigma_{r r}+s=0, \quad \sigma_{r \theta}=0, \quad \sigma_{r z}=0, \quad s=0, \quad \text { at } \quad r=r_{1} \quad \text { and } \quad r=r_{2},
$$

while the boundary conditions for free vibrations in the case of an impervious surface are

$$
\sigma_{r r}+s=0, \quad \sigma_{r \theta}=0, \quad \sigma_{r z}=0, \quad \frac{\partial s}{\partial r}=0, \quad \text { at } \quad r=r_{1} \quad \text { and } \quad r=r_{2} \text {. }
$$

Equations (3.16)-(3.19) together with Eq.(4.1) yield eight homogeneous equations for eight arbitrary constants $C_{1}, C_{2}, C_{3}, C_{4}, A_{3}, B_{3}, A_{1}$, and $B_{1}$. A non-trivial solution can be obtained only when the determinant of the coefficients vanishes. Thus the frequency equation for a pervious surface is

$$
\left|\begin{array}{llllllll}
M_{11}\left(r_{1}\right) & M_{12}\left(r_{1}\right) & M_{13}\left(r_{1}\right) & M_{14}\left(r_{1}\right) & M_{15}\left(r_{1}\right) & M_{16}\left(r_{1}\right) & M_{17}\left(r_{1}\right) & M_{18}\left(r_{1}\right) \\
M_{21}\left(r_{1}\right) & M_{22}\left(r_{1}\right) & M_{23}\left(r_{1}\right) & M_{24}\left(r_{1}\right) & M_{25}\left(r_{1}\right) & M_{26}\left(r_{1}\right) & M_{27}\left(r_{1}\right) & M_{28}\left(r_{1}\right) \\
M_{31}\left(r_{1}\right) & M_{32}\left(r_{1}\right) & M_{33}\left(r_{1}\right) & M_{34}\left(r_{1}\right) & M_{35}\left(r_{1}\right) & M_{36}\left(r_{1}\right) & M_{37}\left(r_{1}\right) & M_{38}\left(r_{1}\right) \\
M_{41}\left(r_{1}\right) & M_{42}\left(r_{1}\right) & M_{43}\left(r_{1}\right) & M_{44}\left(r_{1}\right) & 0 & 0 & 0 & 0 \\
M_{11}\left(r_{2}\right) & M_{12}\left(r_{2}\right) & M_{13}\left(r_{2}\right) & M_{14}\left(r_{2}\right) & M_{15}\left(r_{2}\right) & M_{16}\left(r_{2}\right) & M_{17}\left(r_{2}\right) & M_{18}\left(r_{2}\right) \\
M_{21}\left(r_{2}\right) & M_{22}\left(r_{2}\right) & M_{23}\left(r_{2}\right) & M_{24}\left(r_{2}\right) & M_{25}\left(r_{2}\right) & M_{26}\left(r_{2}\right) & M_{27}\left(r_{2}\right) & M_{28}\left(r_{2}\right) \\
M_{31}\left(r_{2}\right) & M_{32}\left(r_{2}\right) & M_{33}\left(r_{2}\right) & M_{34}\left(r_{2}\right) & M_{35}\left(r_{2}\right) & M_{36}\left(r_{2}\right) & M_{37}\left(r_{2}\right) & M_{38}\left(r_{2}\right) \\
M_{41}\left(r_{2}\right) & M_{42}\left(r_{2}\right) & M_{43}\left(r_{2}\right) & M_{44}\left(r_{2}\right) & 0 & 0 & 0 & 0
\end{array}\right|=0 .
$$

Similarly, with the help of Eqs (3.16)-(3.18) and (3.20) together with Eq.(4.2) we get the frequency equation of an impervious surface

$$
\left|\begin{array}{llllllll}
M_{11}\left(r_{1}\right) & M_{12}\left(r_{1}\right) & M_{13}\left(r_{1}\right) & M_{14}\left(r_{1}\right) & M_{15}\left(r_{1}\right) & M_{16}\left(r_{1}\right) & M_{17}\left(r_{1}\right) & M_{18}\left(r_{1}\right) \\
M_{21}\left(r_{1}\right) & M_{22}\left(r_{1}\right) & M_{23}\left(r_{1}\right) & M_{24}\left(r_{1}\right) & M_{25}\left(r_{1}\right) & M_{26}\left(r_{1}\right) & M_{27}\left(r_{1}\right) & M_{28}\left(r_{1}\right) \\
M_{31}\left(r_{1}\right) & M_{32}\left(r_{1}\right) & M_{33}\left(r_{1}\right) & M_{34}\left(r_{1}\right) & M_{35}\left(r_{1}\right) & M_{36}\left(r_{1}\right) & M_{37}\left(r_{1}\right) & M_{38}\left(r_{1}\right) \\
N_{41}\left(r_{1}\right) & N_{42}\left(r_{1}\right) & N_{43}\left(r_{1}\right) & N_{44}\left(r_{1}\right) & 0 & 0 & 0 & 0 \\
M_{11}\left(r_{2}\right) & M_{12}\left(r_{2}\right) & M_{13}\left(r_{2}\right) & M_{14}\left(r_{2}\right) & M_{15}\left(r_{2}\right) & M_{16}\left(r_{2}\right) & M_{17}\left(r_{2}\right) & M_{18}\left(r_{2}\right) \\
M_{21}\left(r_{2}\right) & M_{22}\left(r_{2}\right) & M_{23}\left(r_{2}\right) & M_{24}\left(r_{2}\right) & M_{25}\left(r_{2}\right) & M_{26}\left(r_{2}\right) & M_{27}\left(r_{2}\right) & M_{28}\left(r_{2}\right) \\
M_{31}\left(r_{2}\right) & M_{32}\left(r_{2}\right) & M_{33}\left(r_{2}\right) & M_{34}\left(r_{2}\right) & M_{35}\left(r_{2}\right) & M_{36}\left(r_{2}\right) & M_{37}\left(r_{2}\right) & M_{38}\left(r_{2}\right) \\
N_{41}\left(r_{2}\right) & N_{42}\left(r_{2}\right) & N_{43}\left(r_{2}\right) & N_{44}\left(r_{2}\right) & 0 & 0 & 0 & 0
\end{array}\right|=0 .(4.4)
$$

In Eqs (4.3), (4.4) the elements $M_{i j}(r)$ and $N_{i j}(r)$ are defined in Eq.(23). 
By ignoring the liquid effects in the frequency equation of a pervious surface (4.3), the results of a purely elastic solid are obtained as a special case considered by Gazis (1959). The frequency equation of an impervious surface has no counterpart in a purely elastic solid. Now we consider the particular cases of the general frequency Eqs (3.3), (3.4) when the axial and angular wavenumbers vanish.

\subsection{Motion independent of $z$}

When the axial wavenumber $k$ is taken equal to zero, i.e., by considering zeroth azimuthal mode, the frequency Eq.(4.3) of a pervious surface degenerates into the product of two determinants as

$$
D_{1} D_{2}=0
$$

where

$$
\begin{aligned}
D_{1} & =\left|\begin{array}{llllll}
M_{11}\left(r_{1}\right) & M_{12}\left(r_{1}\right) & M_{13}\left(r_{1}\right) & M_{14}\left(r_{1}\right) & M_{15}\left(r_{1}\right) & M_{16}\left(r_{1}\right) \\
M_{21}\left(r_{1}\right) & M_{22}\left(r_{1}\right) & M_{23}\left(r_{1}\right) & M_{24}\left(r_{1}\right) & M_{25}\left(r_{1}\right) & M_{26}\left(r_{1}\right) \\
M_{41}\left(r_{1}\right) & M_{42}\left(r_{1}\right) & M_{43}\left(r_{1}\right) & M_{44}\left(r_{1}\right) & 0 & 0 \\
M_{11}\left(r_{2}\right) & M_{12}\left(r_{2}\right) & M_{13}\left(r_{2}\right) & M_{14}\left(r_{2}\right) & M_{15}\left(r_{2}\right) & M_{16}\left(r_{2}\right) \\
M_{21}\left(r_{2}\right) & M_{22}\left(r_{2}\right) & M_{23}\left(r_{2}\right) & M_{24}\left(r_{2}\right) & M_{25}\left(r_{2}\right) & M_{26}\left(r_{2}\right) \\
M_{41}\left(r_{2}\right) & M_{42}\left(r_{2}\right) & M_{43}\left(r_{2}\right) & M_{44}\left(r_{2}\right) & 0 & 0
\end{array}\right|, \\
D_{2} & =\left|\begin{array}{lll}
M_{37}\left(r_{1}\right) & M_{38}\left(r_{1}\right) \\
M_{37}\left(r_{2}\right) & M_{38}\left(r_{2}\right)
\end{array}\right| .
\end{aligned}
$$

The terms $M_{i j}(r)$ appearing in $D_{1}$ and $D_{2}$ are given in Eqs (3.21) for $k=0$. It is clear that for $k=0, \alpha_{1}^{2}$, $\alpha_{2}^{2}, \alpha_{3}^{2}$, are all positive thereby Bessel functions of first and second kind enter the solution.

Arguing on similar lines the frequency equation of an impervious surface (4.4) when $k=0$ reduces to the product of two determinants

$$
D_{3} D_{4}=0
$$

where the determinants $D_{3}, D_{4}$ are

$$
\begin{aligned}
D_{3} & =\left|\begin{array}{llllll}
M_{11}\left(r_{1}\right) & M_{12}\left(r_{1}\right) & M_{13}\left(r_{1}\right) & M_{14}\left(r_{1}\right) & M_{15}\left(r_{1}\right) & M_{16}\left(r_{1}\right) \\
M_{21}\left(r_{1}\right) & M_{22}\left(r_{1}\right) & M_{23}\left(r_{1}\right) & M_{24}\left(r_{1}\right) & M_{25}\left(r_{1}\right) & M_{26}\left(r_{1}\right) \\
N_{41}\left(r_{1}\right) & N_{42}\left(r_{1}\right) & N_{43}\left(r_{1}\right) & N_{44}\left(r_{1}\right) & 0 & 0 \\
M_{11}\left(r_{2}\right) & M_{12}\left(r_{2}\right) & M_{13}\left(r_{2}\right) & M_{14}\left(r_{2}\right) & M_{15}\left(r_{2}\right) & M_{16}\left(r_{2}\right) \\
M_{21}\left(r_{2}\right) & M_{22}\left(r_{2}\right) & M_{23}\left(r_{2}\right) & M_{24}\left(r_{2}\right) & M_{25}\left(r_{2}\right) & M_{26}\left(r_{2}\right) \\
N_{41}\left(r_{2}\right) & N_{42}\left(r_{2}\right) & N_{43}\left(r_{2}\right) & N_{44}\left(r_{2}\right) & 0 & 0
\end{array}\right|, \\
D_{4} & =\left|\begin{array}{lllll}
M_{37}\left(r_{1}\right) & M_{38}\left(r_{1}\right) \\
M_{37}\left(r_{2}\right) & M_{38}\left(r_{2}\right)
\end{array}\right| .
\end{aligned}
$$

Equation (4.5) is satisfied, if either $D_{1}$ or $D_{2}$ is equal to zero. The case of $D_{l}=0$ corresponds to planestrain vibrations of thick-walled hollow poroelastic cylinders, discussed by Malla Reddy and Tajuddin 
(2000), for a pervious surface. Equation (4.7) is satisfied when either $D_{3}=0$, or $D_{4}=0$. The case of $D_{3}=0$, corresponds to the plane-strain vibrations of thick-walled hollow poroelastic cylinders for an impervious surface discussed by Malla Reddy and Tajuddin (2000). From Eqs (4.6) and (4.8) it is clear that $D_{2}$ and $D_{4}$ are same. The case of $D_{2}=0$, corresponds to longitudinal shear vibrations which involve only longitudinal displacement w. Also, since $D_{2}=0$, is the same for a pervious and an impervious surface, hence we find that longitudinal shear vibrations are independent of the nature of the surface. From Eqs (4.5) and (4.7) we find that plane-strain vibrations and longitudinal shear vibrations are uncoupled for a pervious and an impervious surface, respectively, when the motion is independent of the longitudinal coordinate $z$, and these vibrations are coupled for the non-zero longitudinal wavenumber $k$, that is $k \neq 0$. The frequency equation of longitudinal shear vibrations $D_{2}=0$, when expanded gives

$$
J_{n}^{\prime}\left(\alpha_{3} r_{1}\right) Y_{n}^{\prime}\left(\alpha_{3} r_{2}\right)-J_{n}^{\prime}\left(\alpha_{3} r_{2}\right) Y_{n}^{\prime}\left(\alpha_{3} r_{1}\right)=0
$$

and the amplitude ratio is given as

$$
\frac{A_{1}}{B_{1}}=-\frac{Y_{n}^{\prime}\left(\alpha_{3} r_{1}\right)}{J_{n}^{\prime}\left(\alpha_{3} r_{1}\right)} .
$$

The frequency equation of longitudinal shear vibrations of hollow poroelastic cylinders (4.9) is discussed by Tajuddin and Ahmed Shah (2010a).

\subsection{Motion independent of $\theta$}

When the motion is independent of the angular coordinate $\theta$ (i.e., $n=0$ ), the frequency Eq.(4.3) for a pervious surface is reduced to the product of two determinants given by

$$
D_{5} D_{6}=0
$$

where

$$
\begin{aligned}
D_{5} & =\left|\begin{array}{llllll}
M_{11}\left(r_{1}\right) & M_{12}\left(r_{1}\right) & M_{13}\left(r_{1}\right) & M_{14}\left(r_{1}\right) & M_{17}\left(r_{1}\right) & M_{18}\left(r_{1}\right) \\
M_{31}\left(r_{1}\right) & M_{32}\left(r_{1}\right) & M_{33}\left(r_{1}\right) & M_{34}\left(r_{1}\right) & M_{37}\left(r_{1}\right) & M_{38}\left(r_{1}\right) \\
M_{41}\left(r_{1}\right) & M_{42}\left(r_{1}\right) & M_{43}\left(r_{1}\right) & M_{44}\left(r_{1}\right) & 0 & 0 \\
M_{11}\left(r_{2}\right) & M_{12}\left(r_{2}\right) & M_{13}\left(r_{2}\right) & M_{14}\left(r_{2}\right) & M_{17}\left(r_{2}\right) & M_{18}\left(r_{2}\right) \\
M_{31}\left(r_{2}\right) & M_{32}\left(r_{2}\right) & M_{33}\left(r_{2}\right) & M_{34}\left(r_{2}\right) & M_{37}\left(r_{2}\right) & M_{38}\left(r_{2}\right) \\
M_{41}\left(r_{2}\right) & M_{42}\left(r_{2}\right) & M_{43}\left(r_{2}\right) & M_{44}\left(r_{2}\right) & 0 & 0
\end{array}\right|, \\
D_{6} & =\left|\begin{array}{lll}
M_{25}\left(r_{1}\right) & M_{26}\left(r_{1}\right) \\
M_{25}\left(r_{2}\right) & M_{26}\left(r_{2}\right)
\end{array}\right| .
\end{aligned}
$$

The terms $M_{i j}(r)$ in $D_{5}$ and $D_{6}$ are given by Eqs (3.21) for $n=0$.

Similarly, the frequency equation for an impervious surface (4.4), for $n=0$ yields into the product of two determinants as

$$
D_{7} D_{8}=0 \text {, }
$$

where the determinants $D_{7}, D_{8}$ are 


$$
\begin{aligned}
D_{7} & =\left|\begin{array}{llllll}
M_{11}\left(r_{1}\right) & M_{12}\left(r_{1}\right) & M_{13}\left(r_{1}\right) & M_{14}\left(r_{1}\right) & M_{17}\left(r_{1}\right) & M_{18}\left(r_{1}\right) \\
M_{31}\left(r_{1}\right) & M_{32}\left(r_{1}\right) & M_{33}\left(r_{1}\right) & M_{34}\left(r_{1}\right) & M_{37}\left(r_{1}\right) & M_{38}\left(r_{1}\right) \\
N_{41}\left(r_{1}\right) & N_{42}\left(r_{1}\right) & N_{43}\left(r_{1}\right) & N_{44}\left(r_{1}\right) & 0 & 0 \\
M_{11}\left(r_{2}\right) & M_{12}\left(r_{2}\right) & M_{13}\left(r_{2}\right) & M_{14}\left(r_{2}\right) & M_{17}\left(r_{2}\right) & M_{18}\left(r_{2}\right) \\
M_{31}\left(r_{2}\right) & M_{32}\left(r_{2}\right) & M_{33}\left(r_{2}\right) & M_{34}\left(r_{2}\right) & M_{37}\left(r_{2}\right) & M_{38}\left(r_{2}\right) \\
N_{41}\left(r_{2}\right) & N_{42}\left(r_{2}\right) & N_{43}\left(r_{2}\right) & N_{44}\left(r_{2}\right) & 0 & 0
\end{array}\right|, \\
D_{8} & =\left|\begin{array}{lll}
M_{25}\left(r_{1}\right) & M_{26}\left(r_{1}\right) \\
M_{25}\left(r_{2}\right) & M_{26}\left(r_{2}\right)
\end{array}\right| .
\end{aligned}
$$

From Eqs (4.12) and (4.14) it is clear that the determinants $D_{6}$ and $D_{8}$ are same. Now Eq.(4.11) is satisfied if $D_{5}=0$ or $D_{6}=0$. The case of $D_{5}=0$ gives the frequency equation of axially symmetric vibrations of an infinite hollow poroelastic cylinder for a pervious surface discussed by Ahmed Shah (2008). Similarly, Eq.(4.13) is satisfied when $D_{7}=0$ or $D_{8}=0$. The case of $D_{7}=0$ gives the frequency equation of axially symmetric vibrations of an infinite hollow poroelastic cylinder for an impervious surface discussed by Ahmed Shah (2008). The case of $D_{6}=0$ or $D_{8}=0$ when simplified yields the equation

$$
J_{2}\left(\alpha_{3} r_{1}\right) Y_{2}\left(\alpha_{3} r_{2}\right)-J_{2}\left(\alpha_{3} r_{2}\right) Y_{2}\left(\alpha_{3} r_{1}\right)=0 \text {, }
$$

which is the frequency of torsional vibrations of infinite hollow poroelastic cylinders studied by Tajuddin and Ahmed Shah (2007) in the presence of dissipation. Moreover, the frequency Eq.(4.15) is independent of the nature of the surface, i.e., pervious or impervious. Also, Eq.(4.15) is the same for pervious and impervious surfaces.

\subsection{Equivoluminal modes}

For $n=0$, the stress free boundary conditions at the inner and outer surfaces of the hollow poroelastic cylinder are satisfied if

$$
\alpha_{3}^{2}=k^{2}>0 \text {. }
$$

The dilatational and equivoluminal potentials $f_{l}$ and $g_{l}$ are coupled through the boundary conditions. To consider purely equivoluminal modes, we set

$$
f_{1}=f_{2}=0, \quad g_{3}=0
$$

Substituting Eqs (4.17) into Eq.(3.15), it is seen that the dilatations of solid and liquid media are zero. Hence from Eq.(2.2), the liquid pressure is zero. Therefore the equivoluminal modes are independent of the nature of the surface, i.e., pervious and impervious. Accordingly, no distinction between pervious and impervious surfaces is seen. Therefore from Eqs (4.16) and (4.17), we have

where

$$
g_{l}^{\prime}\left(\alpha_{3} r_{l}\right)=g_{l}^{\prime}\left(\alpha_{3} r_{2}\right)=0, \quad\left[\sigma_{r r}+s=0\right]
$$

$$
g_{l}\left(\alpha_{3} r\right)=A_{l} J_{l}\left(\alpha_{3} r\right)+B_{l} Y_{l}\left(\alpha_{3} r\right) .
$$

Substituting Eq.(4.19) into Eq.(4.18) and eliminating the constants $A_{1}, B_{l}$, the frequency equation of purely equivoluminal modes is 


$$
J_{1}^{\prime}\left(\alpha_{3} r_{1}\right) Y_{1}^{\prime}\left(\alpha_{3} r_{2}\right)-J_{1}^{\prime}\left(\alpha_{3} r_{2}\right) Y_{1}^{\prime}\left(\alpha_{3} r_{1}\right)=0
$$

with the amplitude ratio

$$
\frac{A_{1}}{B_{1}}=-\frac{Y_{l}^{\prime}\left(\alpha_{3} r_{1}\right)}{J_{l}^{\prime}\left(\alpha_{3} r_{1}\right)}
$$

and the non-zero displacement and stress for equivoluminal modes are

$$
\begin{aligned}
& u=i k\left[A_{1} J_{l}\left(\alpha_{3} r\right)+B_{1} Y_{l}\left(\alpha_{3} r\right)\right] e^{i(k z+\omega t)}, \quad w=-\alpha_{3}\left[A_{l} J_{0}\left(\alpha_{3} r\right)+B_{1} Y_{0}\left(\alpha_{3} r\right)\right] e^{i(k z+\omega t)}, \\
& \sigma_{r r}=-2 N i k\left[A_{l} J_{l}^{\prime}\left(\alpha_{3} r\right)+B_{1} Y_{l}^{\prime}\left(\alpha_{3} r\right)\right] e^{i(k z+\omega t)} .
\end{aligned}
$$

Equation (4.20) is same as Eq.(4.9) of longitudinal shear vibrations for a case of $n=1$. The frequency Eq.(4.20) for the two limiting values of $h / r_{l}$ is given below:

\section{3.(i) For a thin poroelastic cylindrical shell}

When $h / r_{1}<<1$, that is for a thin poroelastic cylindrical shell, the frequency Eq.(4.20) by using Hankel-Kirchoff asymptotic approximations (Abramowitz and Stegun, 1965) is reduced to

$$
\sin \left(\alpha_{3} h\right)-\frac{7 \alpha_{3} h}{8 \alpha_{3}^{2} r_{1} r_{2}} \cos \left(\alpha_{3} h\right)+\frac{49}{64 \alpha_{3}^{2} r_{1} r_{2}} \sin \left(\alpha_{3} h\right) \approx 0 .
$$

As $\alpha_{3} r_{1} \rightarrow \infty, \alpha_{3} r_{2} \rightarrow \infty$, with the help of Eq.(4.16), Eq.(4.23) is simplified to

$$
\omega \approx \sqrt{2} \frac{q \pi V_{3}}{h}\left[1+\frac{7}{8 q^{2} \pi^{2}}\left(\frac{h}{r_{l}}\right)^{2}\right] . \quad(q=1,2,3, \ldots . .)
$$

Equation (4.24) determines the frequency of purely equivoluminal modes of a poroelastic plate of thickness $h$.

\section{3.(ii) For a poroelastic non-hollow cylinder}

When $r_{l} / h \rightarrow 0$, that is for a poroelastic non-hollow cylinder, the frequency Eq.(4.20) is reduced asymptotically to

$$
J_{1}^{\prime}\left(\alpha_{3} h\right)=0,
$$

which is the frequency equation of purely equivoluminal modes of a poroelastic solid cylinder of radius $h$.

\section{Non-dimensionalisation of frequency equation}

For propagating modes in a non-dissipative medium, the wavenumber $k$ is real. To analyze the frequency equations of pervious and impervious surfaces, it is convenient to introduce the following nondimensional parameters 


$$
\begin{aligned}
& a_{1}=P H^{-1}, \quad a_{2}=Q H^{-1}, \quad a_{3}=R H^{-1}, \quad a_{4}=N H^{-1}, \\
& m_{11}=\rho_{11} \rho^{-1}, \quad m_{12}=\rho_{12} \rho^{-1}, \quad m_{22}=\rho_{22} \rho^{-1}, \quad \Omega=\omega h C_{0}^{-1}, \\
& \tilde{x}=\left(V_{0} V_{1}^{-1}\right)^{2}, \quad \tilde{y}=\left(V_{0} V_{2}^{-1}\right)^{2}, \quad \tilde{z}=\left(V_{0} V_{3}^{-1}\right)^{2}, \quad \delta=h L^{-1}
\end{aligned}
$$

where $\Omega$ is the non-dimensional frequency, $H=P+2 Q+R, \rho=\rho_{11}+2 \rho_{12}+\rho_{22}, C_{0}$ and $V_{0}$ are the reference velocities $\left(C_{0}{ }^{2}=N / \rho, V_{0}{ }^{2}=H / \rho\right), h$ is the thickness of the hollow poroelastic cylinder and $L$ is the wavelength. Let

$$
g=\frac{r_{2}}{r_{1}} \quad \text { so that } \quad \frac{h}{r_{1}}=(g-1) \text {. }
$$

\section{Numerical results and discussion}

Two types of poroelastic materials are used to carry out the computational work, one is sandstone saturated with kerosene say Material-I, (Fatt, 1959), the other one is sandstone saturated with water, Material-II (Yew and Jogi, 1976), whose non-dimensional physical parameters are given in Tab.2

Table 2

\begin{tabular}{|c|c|c|c|c|c|c|c|c|c|c|}
\hline $\begin{array}{c}\text { Material/ } \\
\text { Parameter }\end{array}$ & $a_{1}$ & $a_{2}$ & $a_{3}$ & $a_{4}$ & $m_{11}$ & $m_{12}$ & $m_{22}$ & $\tilde{x}$ & $\tilde{y}$ & $\tilde{z}$ \\
\hline $\mathrm{I}$ & 0.843 & 0.065 & 0.028 & 0.234 & 0.901 & -0.001 & 0.101 & 0.999 & 4.763 & 3.851 \\
\hline $\mathrm{II}$ & 0.960 & 0.006 & 0.028 & 0.412 & 0.877 & 0 & 0.123 & 0.913 & 4.347 & 2.129 \\
\hline
\end{tabular}

For a given poroelastic material, the frequency Eqs (4.3) and (4.4) of pervious and impervious surfaces when non-dimensionalised by using the Eqs (5.1) and (5.2) present the relation between the nondimensional frequency $\Omega$ and ratio of thickness to wavelength $(\delta)$ for given dimensions of the hollow poroelastic cylinder. The ratio of the outer radius to that of the inner radius of the hollow poroelastic cylinder, that is, $g$ takes the values $1.034,3$ and $\infty$, which represent the thin poroelastic shell, thick poroelastic shell and poroelastic non-hollow cylinder, respectively. The number of waves around the circumference, or angular wavenumber $n$ take values 1 and 2 . The value $n=1$ corresponds to flexural vibrations and $n=2$ corresponds to typical non-axially symmetric vibrations.

The non-dimensional frequency $\Omega$ as a function of the ratio of thickness to wavelength $\delta$ is computed for the referred material. The frequencies of first five modes are shown in the figures. The frequencies of the thin poroelastic shell of material-I are presented in Fig.1. for $n=1$ and $n=2$. Frequencies are same for $n=1$ and $n=2$, each for a pervious surface and an impervious surface in the case of a thin poroelastic shell. From Fig. 1 it is clear that for material-I, the frequency of a pervious surface and an impervious surface is same for the first three modes in $0.1 \leq \delta \leq 0.7$, while for the fourth and fifth mode the frequency is same in $0.1<\delta \leq 0.5$ and $0.8<\delta \leq 1$. In $0<\delta \leq 0.1$, the frequency of an impervious surface is more than of a pervious surface for the second, third, fourth and fifth mode while the frequency of an impervious surface is less than the frequency of a pervious surface in $0.7 \leq \delta \leq 1$ for the first mode. The frequencies of the thick poroelastic shell of materialI with $n=1$ is presented in Fig.2. The frequency of an impervious surface is less than that of a pervious surface for the second, third, fourth and fifth mode in $0<\delta<0.1$ and $0.0<\delta \leq 1$, while for the first mode the frequency of an impervious surface is less than that of a pervious surface in $0.3<\delta<0.5$. The frequency of the 
poroelastic non-hollow cylinder of material-I with $n=1$ is presented in Fig.3. The frequency of an impervious surface is more than that of a pervious surface for the third, fourth and fifth mode, while the first and second mode have frequency less than that of a pervious surface in $0.1<\delta \leq 0.3$.

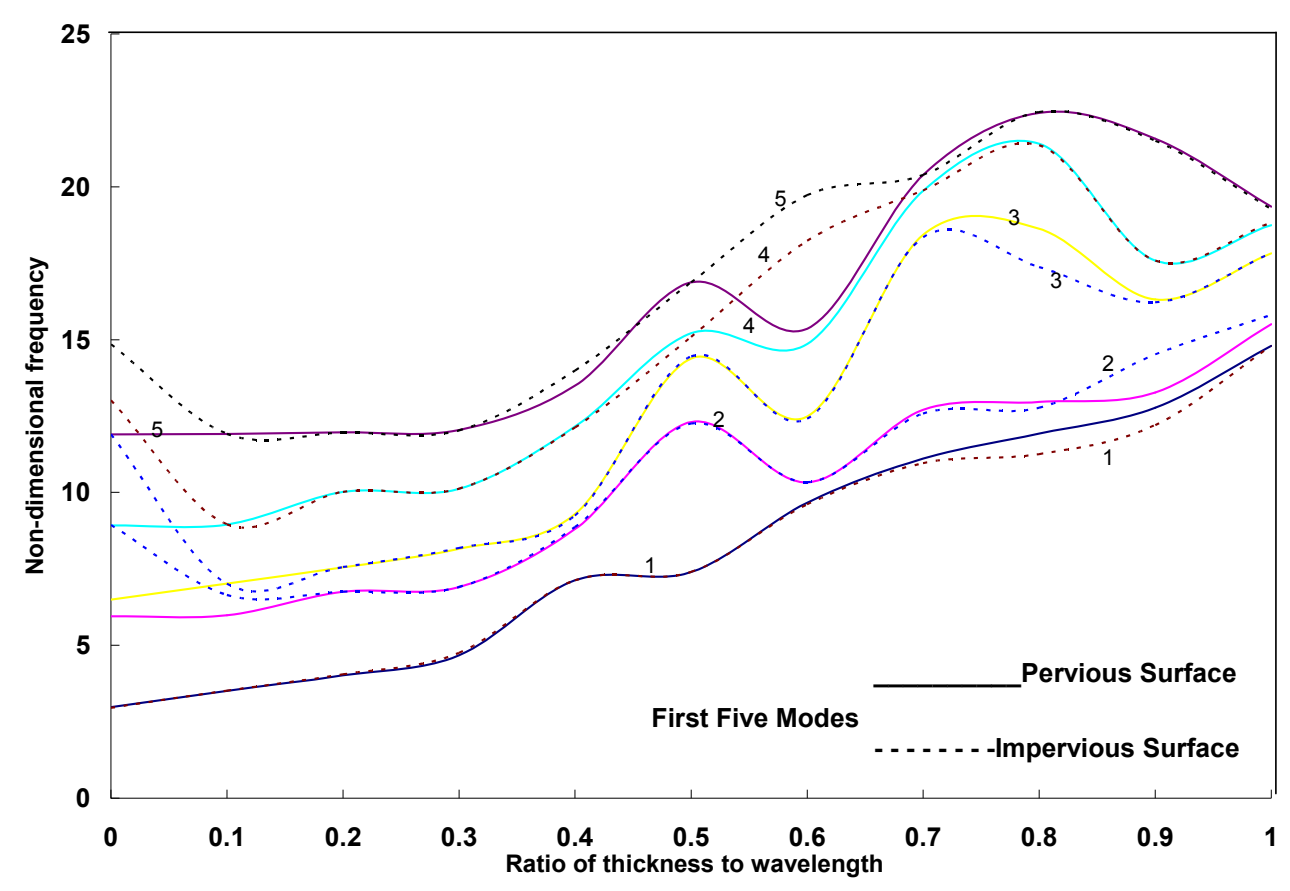

Fig.1. Frequency as a function of wavelength (Mat - I, thin-shell, $n=1,2$ ).

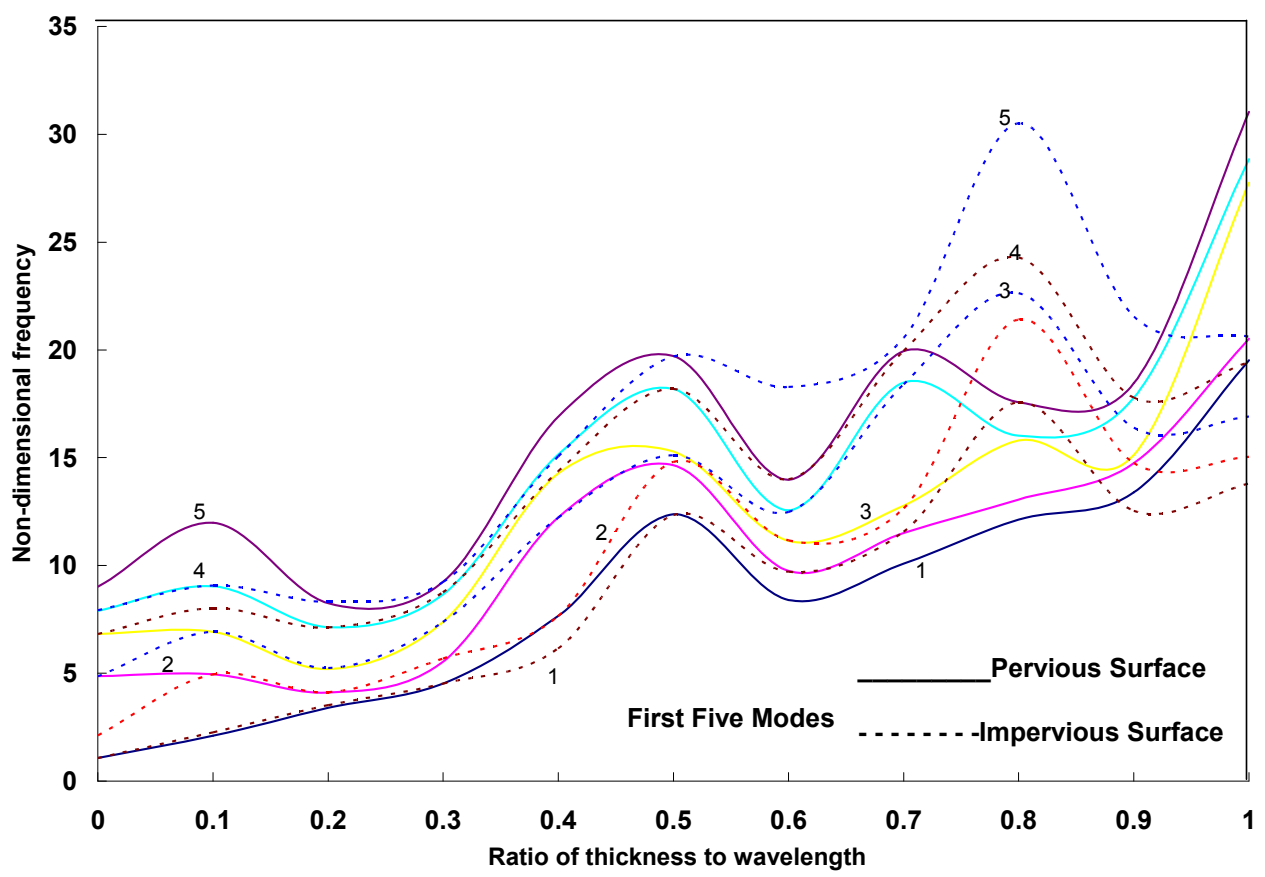

Fig.2. Frequency as a function of wavelength (Mat $-\mathrm{I}$, thick-shell, $n=1$ ). 


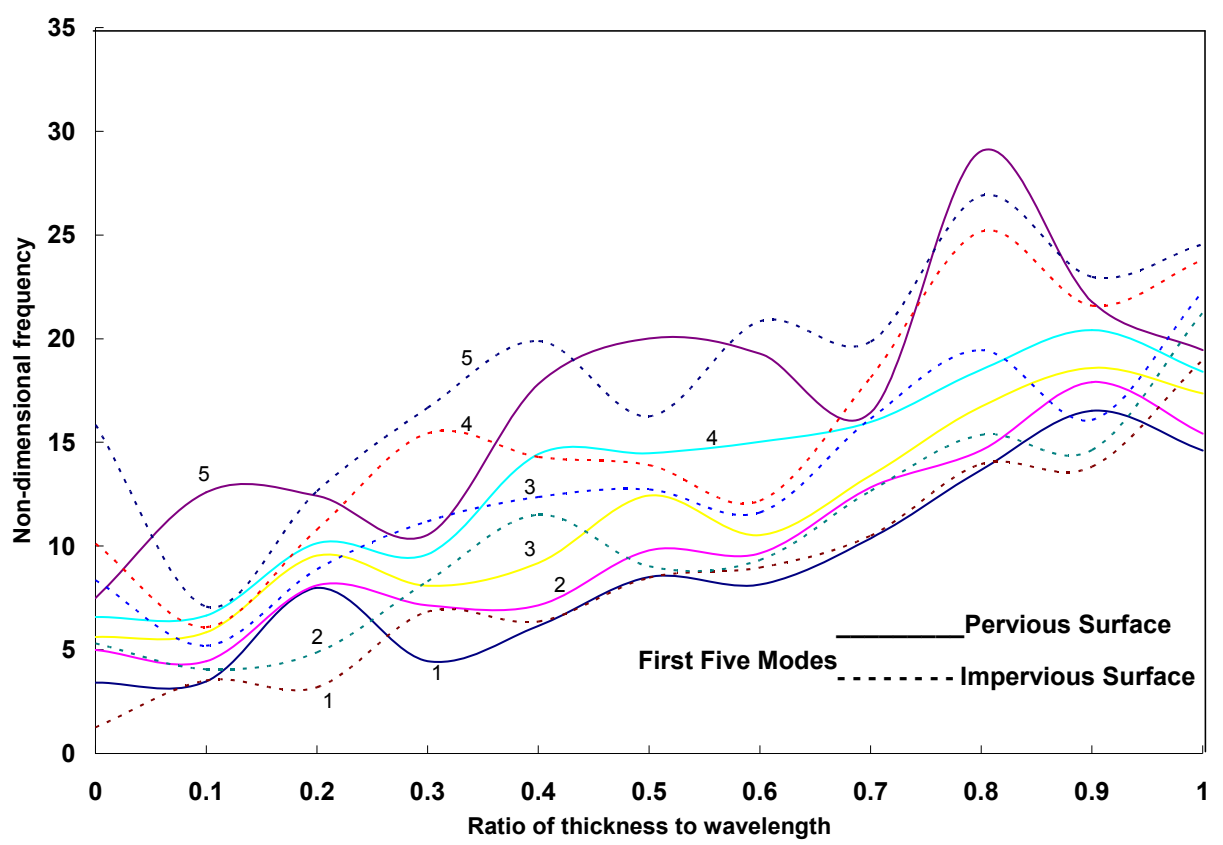

Fig.3. Frequency as a function of wavelength (Mat $-\mathrm{I}$, non-hollow cylinder, $n=1$ ).

The frequencies of the thick poroelastic shell of material-I when $n=2$ are presented in Fig.4. The frequency of a pervious and an impervious surface is same in $0.3<\delta<0.8$ for the first five modes. In $0<\delta<0.3$, the frequency of an impervious surface is less than that of a pervious surface for the first, fourth and fifth mode. In $0.8<\delta<1$, the frequency of an impervious surface is less than that of a pervious surface for the first five modes. The frequency of the poroelastic solid cylinder of material-I with $n=2$ is shown in Fig.5. The frequency of an impervious surface in the case of the third, fourth and fifth mode is less than that of corresponding modes of a pervious surface in $0.5<\delta \leq 1$. The modes near the origin, when the wavelength is large are presented for material-I in Fig.6. From Fig. 6 it is observed that the frequency of an impervious surface is more than that of a pervious surface for $n=1$ and $n=2$. Also, these frequencies are constant.

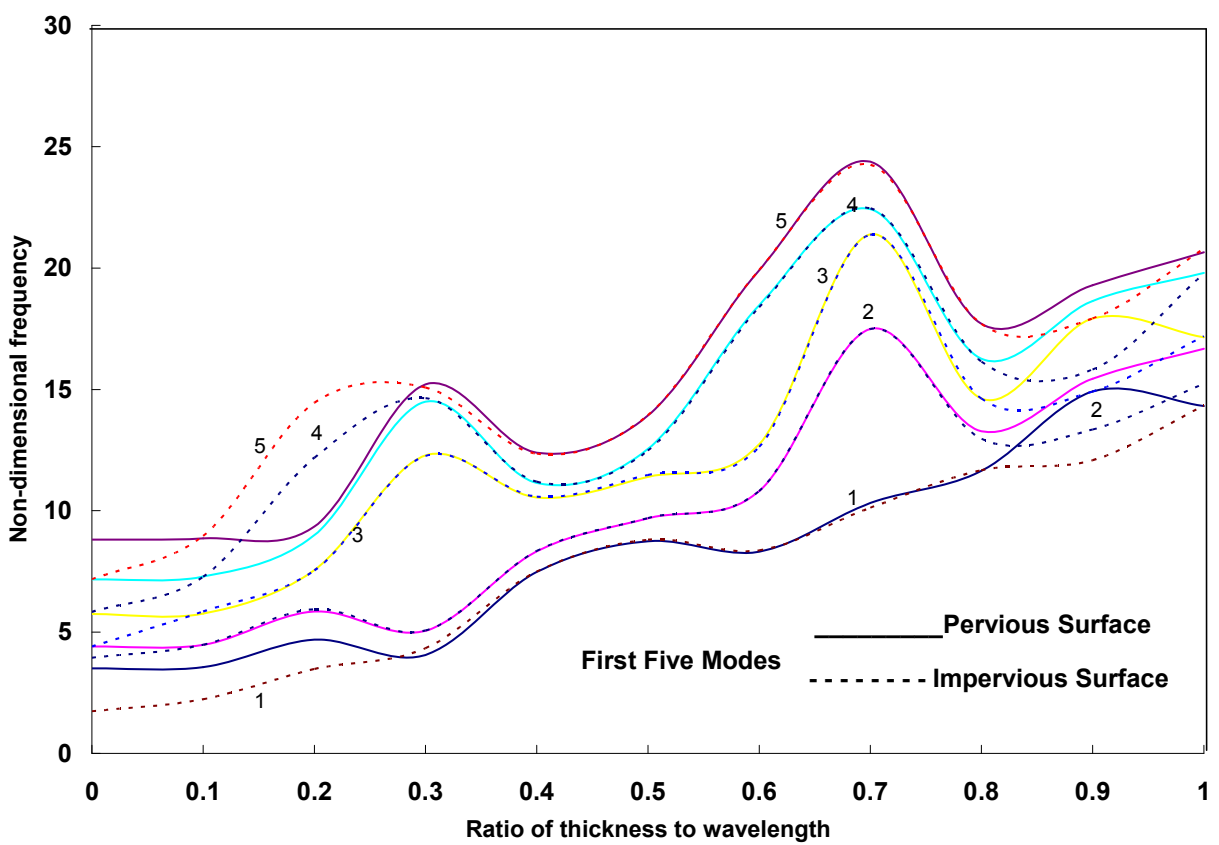

Fig.4. Frequency as a function of wavelength (Mat $-\mathrm{I}$, thich-shell, $n=2$ ). 




Fig.5. Frequency as a function of wavelength (Mat $-\mathrm{I}$, non-hollow cylinder, $n=2$ ).

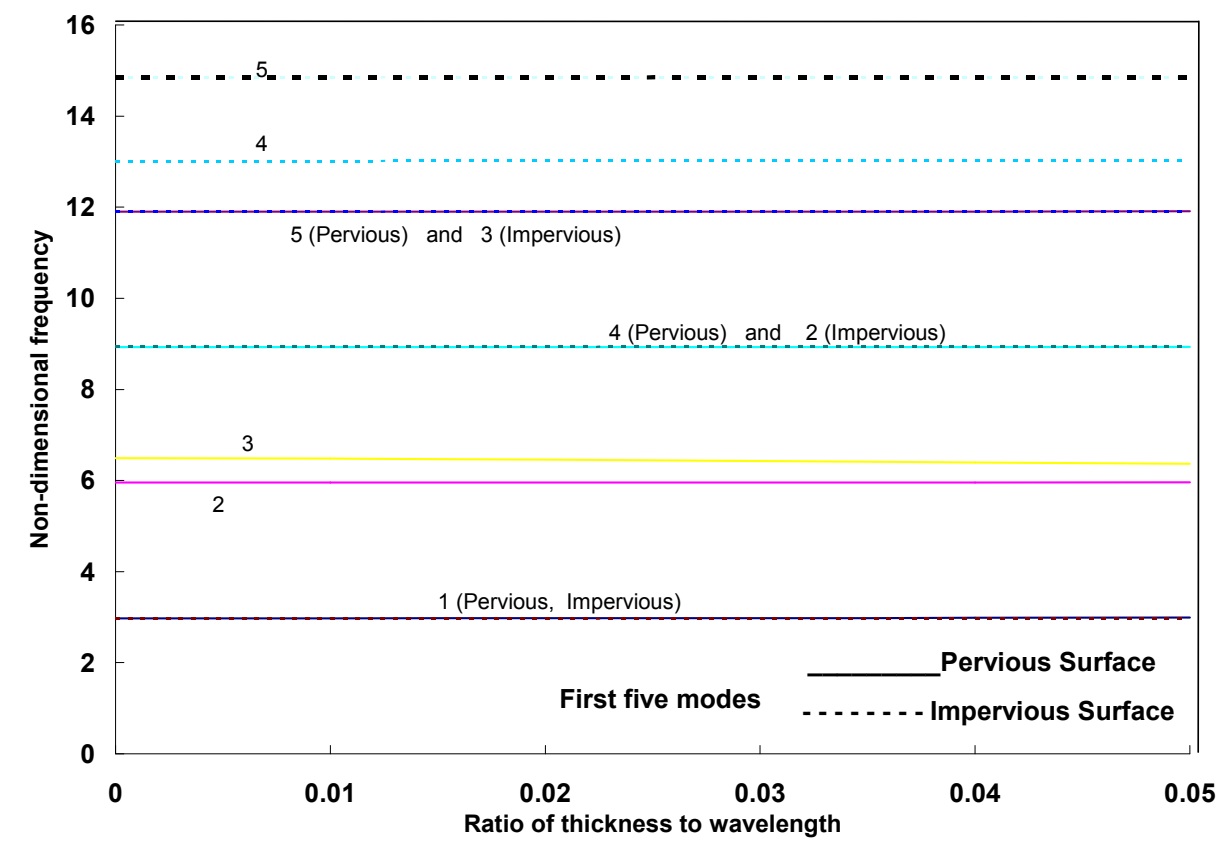

Fig.6. Frequency as a function of wavelength (Mat - I, thin-shell, large wavelength, $n=1,2$ ).

The frequencies of the thin poroelastic shell of material-II are shown in Fig. 7 for $n=1$ and $n=2$. The frequencies are same for $n=1$ and $n=2$ in the case of a thin poroelastic shell. From Fig. 7 it is clear that in $0.3 \leq \delta \leq 0.5$ the frequency of an impervious surface is less than that of a pervious surface for the first five modes while in $0 \leq \delta<0.3$ and $0.5 \leq \delta \leq 1$, the frequency of a pervious and an impervious surfaces are same for all the first five modes. The frequency versus the ratio of thickness to wavelength for a thick poroelastic shell 
with $n=1$ is presented in Fig.8 in the case of material-II. It is seen that the frequency of an impervious surface for all the first five modes is higher than that of a pervious surface in $0.7<\delta<0.9$. The frequency of an impervious surface is less than that of a pervious surface in $0.2<\delta<0.4$, and same in the case of the first three modes in $0.4<\delta \leq 1$. The fourth and fifth mode have same frequency for a pervious and an impervious surface in $0.6 \leq \delta \leq 1$. In $0.4<\delta<0.6$, the frequency of an impervious surface is more for the fourth and fifth mode than the corresponding modes of a pervious surface. It is observed from Fig.9 that for the poroelastic solid cylinder of material-II, the frequency of the first five modes of an impervious surface is less than corresponding modes of a pervious surface in $0.8<\delta<0.9$. The frequency of the second, third, fourth and fifth mode of an impervious surface is less than that of the corresponding modes of a pervious surface in $0.1<\delta<0.3$, while the frequency of an impervious surface for the first mode is less than that of a pervious surface in $0.65 \leq \delta \leq 0.75$. The frequency of the thick poroelastic shell of material-II is shown in Fig. 10 for $n=2$. It is observed that in $0<\delta<0.3$, the frequency of an impervious surface is more than that of a pervious surface for the first five modes, just an opposite phenomenon to material-I. The frequency of a pervious surface and an impervious surface is same in $0.3<\delta<0.6$ and $0.8<\delta<1$ for all the first five modes. In $0.6<\delta<0.8$, the frequency of an impervious surface is more than that of a pervious surface.

The variation of frequency of the poroelastic solid cylinder of material-II when $n=2$ is shown in Fig.11. The frequency of an impervious surface is more than that of a pervious surface for the first five modes in $0 \leq \delta \leq 0.3$, and $0.5<\delta \leq 0$. 7. The frequency of an impervious surface of the first five modes is less than that of corresponding modes of a pervious surface in $0.3<\delta \leq 0.5$ and $0.7<\delta \leq 1$. The modes near the origin for material-II with $n=1$ and $n=2$ are presented in Fig.12. From Fig. 12 it is seen that the frequency of a pervious and an impervious surface is same for material-II when $n=1$ and $n=2$. The frequency of material-I is higher than that of material-II for pervious and impervious surfaces.

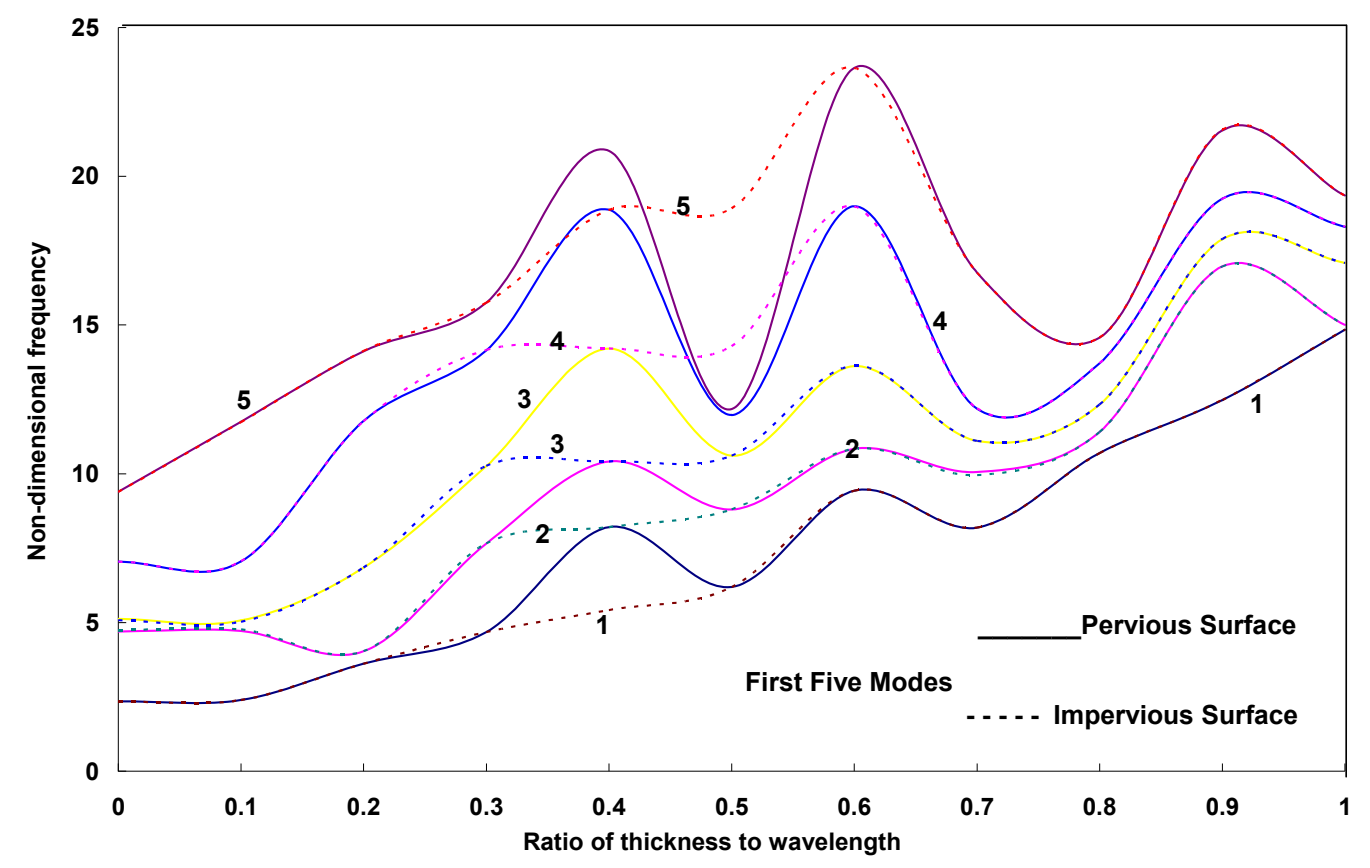

Fig.7. Frequency as a function of wavelength (Mat - II, thin-shell, $n=1,2$ ). 




Fig.8. Frequency as a function of wavelength (Mat - II, thick-shell, $n=1$ ).

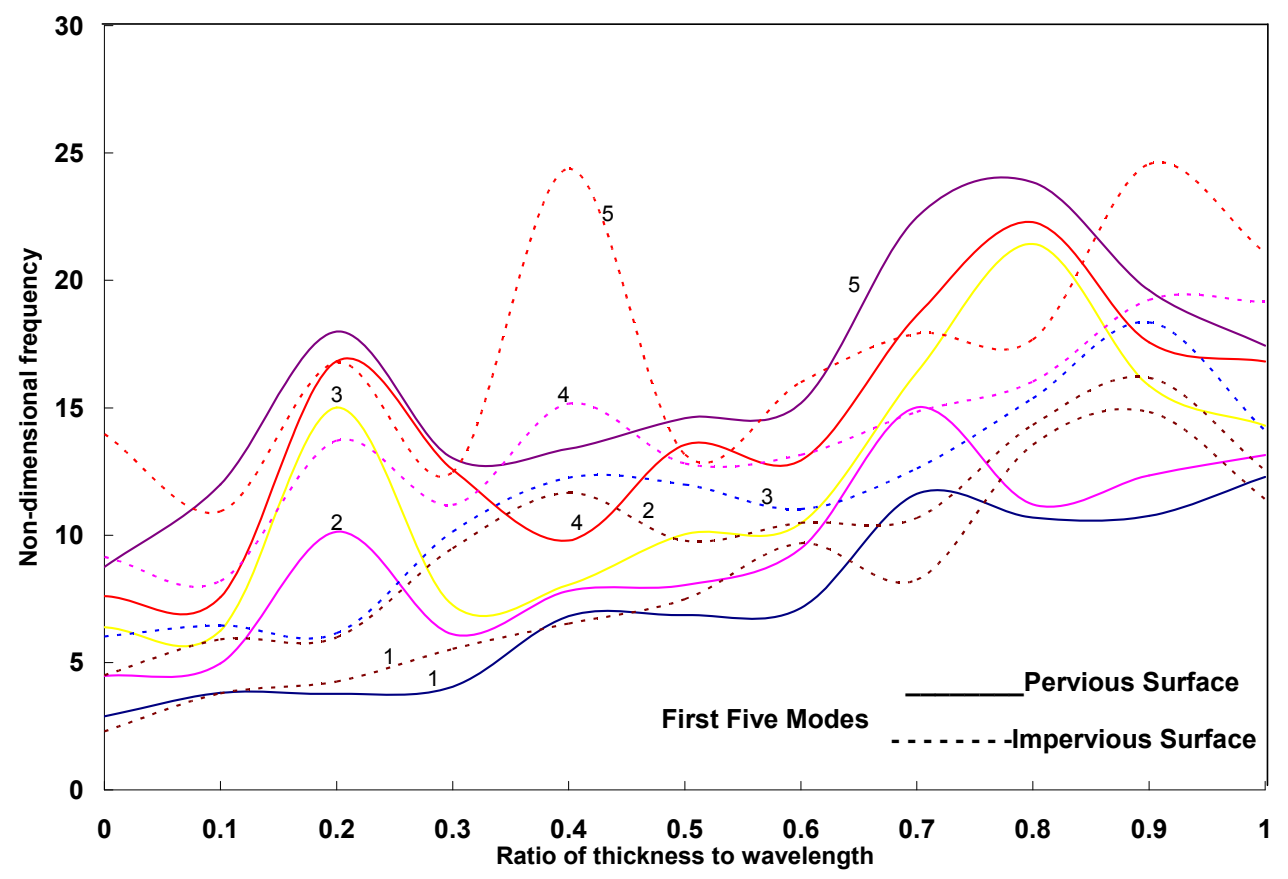

Fig.9. Frequency as a function of wavelength (Mat - II, non-hollow cylinder, $n=1$ ). 


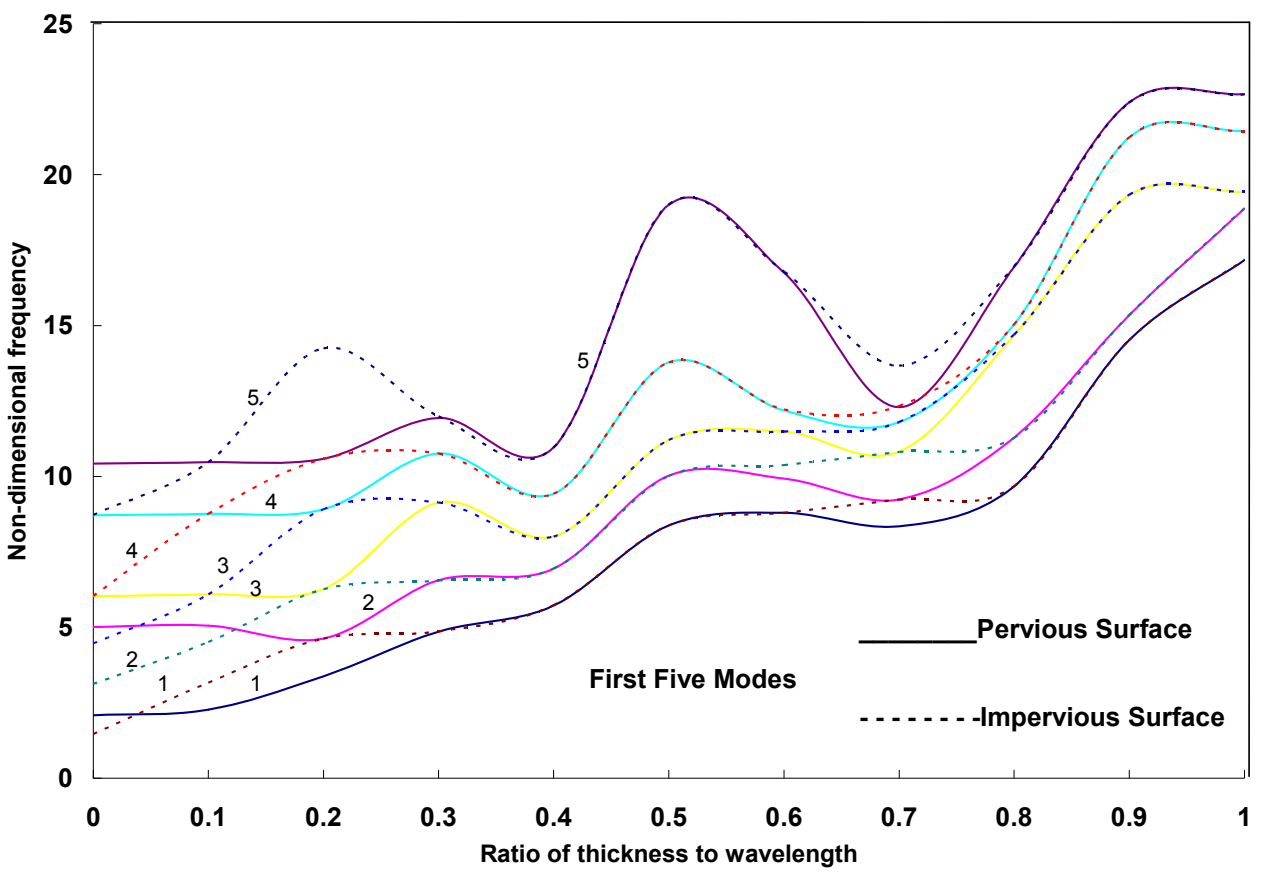

Fig.10. Frequency as a function of wavelength (Mat - II, thick-shell, $n=2$ ).

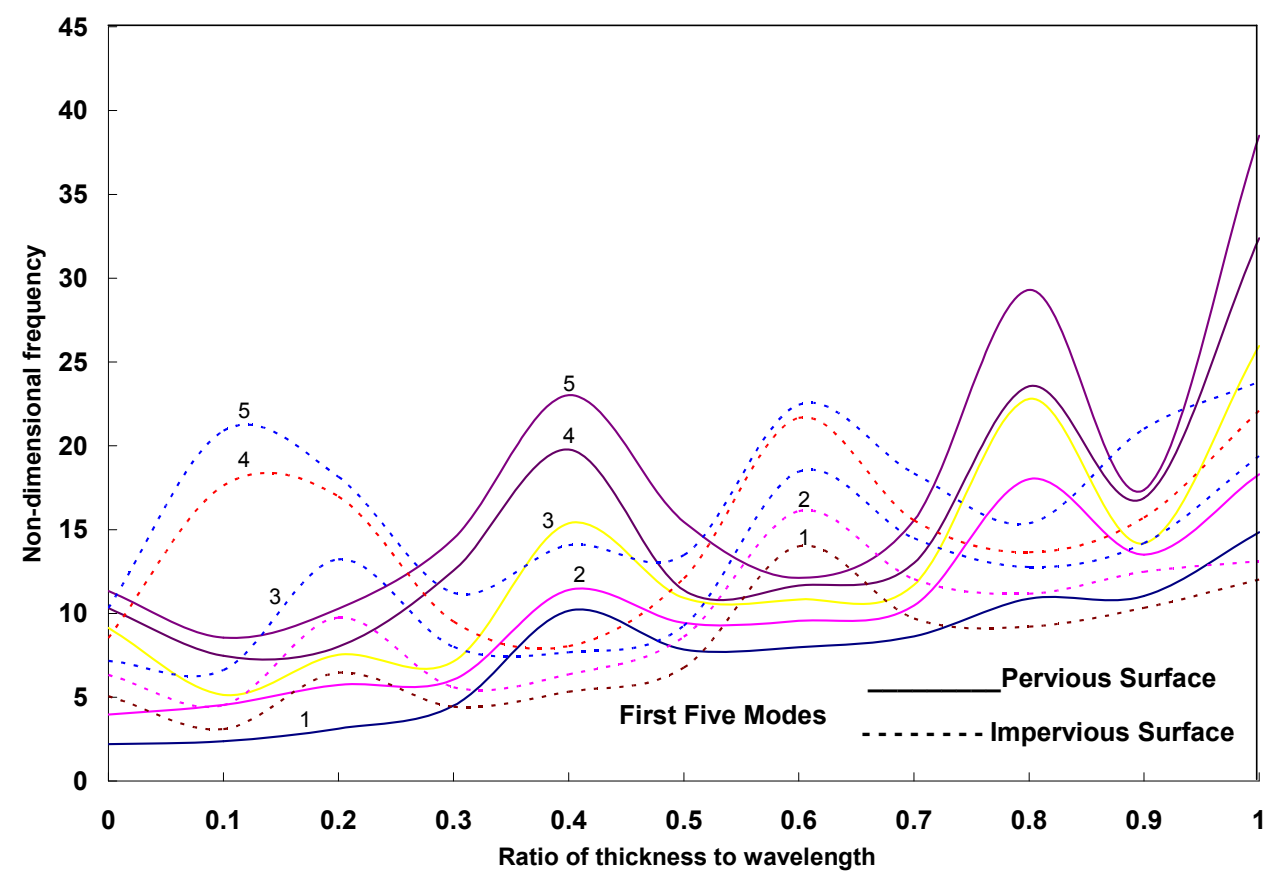

Fig.11. Frequency as a function of wavelength (Mat - II, non-hollow cylinder, $n=2$ ). 




Fig.12. Frequency as a function of wavelength (Mat - II, thin-shell, large wavelength, $n=1,2$ ).

\section{Concluding remarks}

The investigation of the propagation of wave in hollow poroelastic circular cylinders of infinite extent has lead to the following conclusions:

(i) When $n \neq 0$ and $k=0$, non-axially symmetric plane-strain modes are generated and uncoupled family of longitudinal shear vibrations are derived from the potential function $g_{l}(r)$ alone as a special case.

(ii) When $k \neq 0$ and $n=0$ axially symmetric longitudinal modes are generated.

(iii) When both the number of waves around the circumference $\mathrm{n}$ and longitudinal wavenumber $k$ are zero, the motion is axially symmetric and of infinite wavelength. The potential functions $f_{l}(r), g_{l}(r)$ and $g_{3}(r)$ generate three types of uncoupled vibrations that are plane-strain extensional, plane-strain shear and longitudinal shear vibrations.

(iv) For $n \neq 0$ and $k \neq 0$ the potential functions $f_{l}(r), g_{l}(r)$ and $g_{3}(r)$ are coupled through the boundary conditions and generate the non-axially symmetric vibrations.

(v) The frequency is same for flexural and non-axially symmetric vibrations both for a pervious and impervious surface, in the case of a thin poroelastic cylindrical shell. This is not true for a thick poroelastic shell or a poroelastic non-hollow cylinder.

(vi) In general, the frequency of an impervious surface is higher than that of a pervious surface.

(vii) When the wavelength is large, the propagation of waves in thin poroelastic cylindrical shells is nondispersive.

\section{Nomenclature}

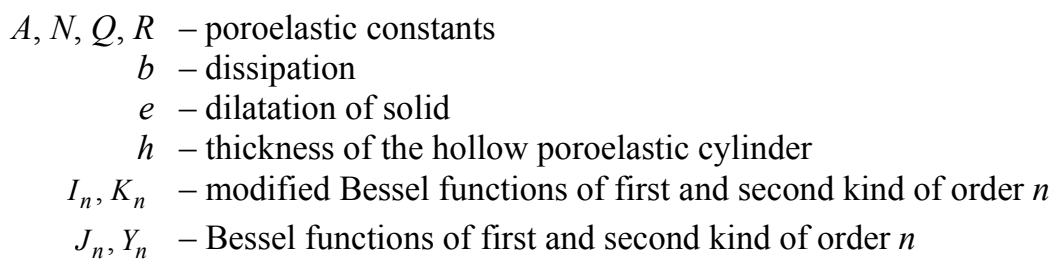




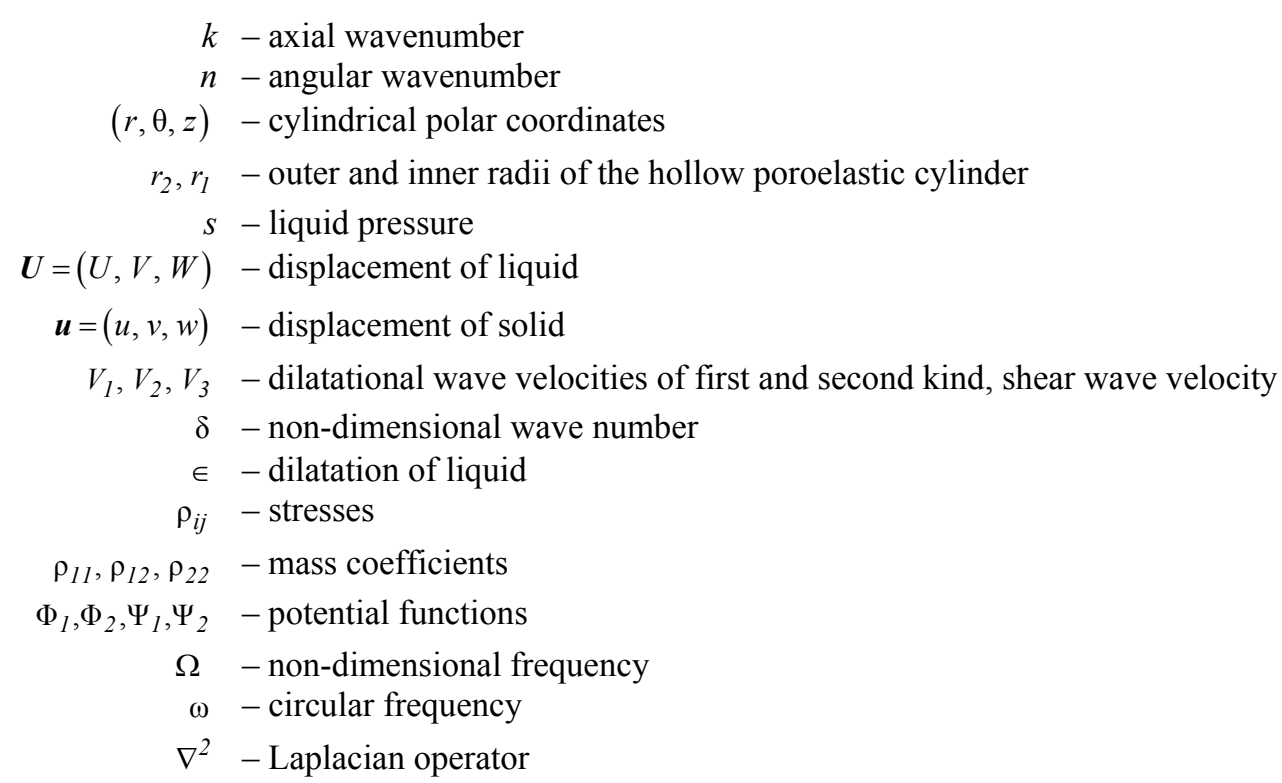

\section{References}

Abramowitz A. and Stegun I.A. (1965): Handbook of Mathematical Functions. - National Bureau of Standards, Washington.

Ahmed Shah S. (2008): Axially symmetric vibrations of fluid-filled poroelastic circular cylindrical shells. - Journal of Sound and Vibration, vol.318, pp.389-405.

Ahmed Shah S. and Tajuddin M. (2011): Torsional vibrations of poroelastic prolate spheroids. - International Journal of Applied Mechanics and Engineering, vol.16, pp.521-529.

Berryman J.G. and Pride S.R. (2005): Dispersion of waves in porous cylinders with patchy saturation: Formulation and torsional waves. - J. Acoust. Soc. Am., vol.117, pp.1785-1795.

Biot M.A. (1956): Theory of propagation of elastic waves in fluid-saturated porous solid. - J. Acoust. Soc. Am., vol.28, pp.168-178.

Chao G., Smeulders D.M.J. and van Dongen M.E.H. (2004): Shock-induced borehole waves in porous formations: Theory and experiments. - J. Acoust. Soc. Am., vol.116, pp.693-702.

Farhang H., Esmaeil E., Anthony N.S. and Mirnezami A. (2007): Wave propagation in transversely isotropic cylinders. - Int. Journal of Solids and Structures, vol.44, pp.5236-5246.

Fatt I. (1959): The Biot-Willis elastic coefficients for a sandstone. - J. Appl. Mech., vol.26, pp.296-297.

Gazis D.C. (1959): Three-dimensional investigation of the propagation of waves in hollow circular cylinders. - J. Acoust. Soc. Am., vol.31, pp.568-578.

Karpfinger F., Gurevich B., Valero H.P., Bakulin A. and Sinha B. (2010): Tube wave signatures in cylindrically layered porous media computed with the spectral method. - Geophysical Journal International, vol.183, pp.1005-1013.

Love A.E.H. (1944): A Treatise on the Mathematical Theory of Elasticity. - New-York: Dover.

Sharma J.N. and Sharma N. (2010): Three-dimensional free vibration analysis of a homogeneous transradially isotropic thermoelastic sphere. - Trans ASME, J. Appl. Mech., vol.77, 021004 (9 pages).

Tajuddin M. and Sarma K.S. (1980): Torsional vibrations of poroelastic cylinders. - Trans. ASME, J. Appl. Mech., vol.47, pp.214-216.

Tajuddin M. and Ahmed Shah S. (2006): Circumferential waves of infinite hollow poroelastic cylinders. - Trans. ASME, J. Appl. Mech., vol.73, pp.705-708. 
Tajuddin M. and Ahmed Shah S. (2007): On torsional vibrations of infinite hollow poroelastic cylinders. - Journal of Mechanics of Materials and Structures, vol.2, pp.189-200.

Tajuddin M. and Ahmed Shah S. (2010a): Longitudinal shear vibrations of hollow poroelastic cylinders. - Bull. Cal. Math. Soc., vol.102, pp.289-298.

Tajuddin M. and Ahmed Shah S. (2010b): Radial vibrations of thick-walled hollow poroelastic cylinders. - Journal of Porous Media., vol.13, pp.307-318.

Wisse C.J., Smeulders D.M.J., van Dongen M.E.H. and Chao G. (2002): Guided wave modes in porous cylinders: Experimental results. - J. Acoust. Soc. Am., vol.112, pp.890-895.

Yew C.H. and Jogi P.N. (1976): Study of wave motions in fluid-saturated porous rocks. - J. Acoust. Soc. Am., vol.60, pp.2-8.

Received: May 8, 2014

Revised: May 28, 2015 